\title{
ON A PROOF OF THE LABASTIDA-MARIÑO-OOGURI-VAFA CONJECTURE
}

\author{
Kefeng Liu and Pan Peng
}

\begin{abstract}
We outline a proof of a remarkable conjecture of Labastida-Mariño-OoguriVafa about certain new algebraic structures of quantum link invariants and the integrality of infinite family of new topological invariants. Our method is based on the cut-and-join analysis and a special rational ring characterizing the structure of the Chern-Simons partition function.
\end{abstract}

\section{Introduction}

For decades, we have witnessed the great development of string theory and its powerful impact on the development of mathematics. There have been a lot of marvelous results revealed by string theory, which deeply relate different aspects of mathematics. All these mysterious relations are connected by a core idea in string theory called "duality". It was found that string theory on Calabi-Yau manifolds provided new insight in geometry of these spaces. The existence of a topological sector of string theory leads to a simplified model in string theory, the topological string theory.

A major problem in topological string theory is how to compute Gromov-Witten invariants. There are two major methods widely used: mirror symmetry in physics and localization in mathematics. Both methods are effective when genus is low while having trouble in dealing with higher genera due to the rapidly growing complexity during computation. However, when the target manifold is Calabi-Yau threefold, large $N$ Chern-Simons/topological string duality opens a new gate to a complete solution of computing Gromov-Witten invariants at all genera.

The study of large $N$ Chern-Simons/topological string duality was originated in physics by an idea that gauge theory should have a string theory explanation. In 1992, Witten 28] related topological string theory of $T^{*} M$ of a three dimensional manifold $M$ to Chern-Simons gauge theory on $M$. In 1998, Gopakumar and Vafa 4 . conjectured that, at large $N$, open topological A-model of $N$ D-branes on $T^{*} S^{3}$ is dual to topological closed string theory on resolved conifold $\mathcal{O}(-1) \oplus \mathcal{O}(-1) \rightarrow \mathbb{P}^{1}$. Later, Ooguri and Vafa 21] showed a picture on how to describe Chern-Simons invariants of a knot by open topological string theory on resolved conifold paired with lagrangian associated with the knot.

Though large $N$ Chern-Simons/topological string duality still remains open, there have been a lot of progress in this direction demonstrating the power of this idea. Even for the simplest knot, the unknot, Mariño-Vafa formular [19, 15, gives a beautiful closed formula for Hodge integral up to three Chern classes of Hodge bundle. Furthermore, using topological vertex theory [1, 16, 13, one is able to compute Gromov-Witten

Received by the editors April 30, 2007. 
invariants of any toric Calabi-Yau threefold by reducing the computation to a gluing algorithm of topological vertex. This thus leads to a closed formula of topological string partition function, a generating function of Gromov-Witten invariants, in all genera for any toric Calabi-Yau threefolds.

On the other hand, after Jones' famous work on polynomial knot invariants, there had been a series of polynomial invariants discovered (for example, 8, 3, 9]), the generalization of which was provided by quantum group theory [25] in mathematics and by Chern-Simons path integral with the gauge group $S U(N)$ [27] in physics.

Based on the large $N$ Chern-Simons/topological string duality, Ooguri and Vafa 21 reformulated knot invariants in terms of new integral invariants capturing the spectrum of M2 branes ending on M5 branes embedded in the resolved conifold. Later, Labastida, Mariño and Vafa [12, 10] refined the analysis of [21] and conjectured the precise integrality structure for open Gromov-Witten invariants. This conjecture predicts a remarkable new algebraic structure for the generating series of general link invariants and the integrality of infinite family of new topological invariants. In string theory, this is a striking example that two important physical theories, topological string theory and Chern-Simons theory, exactly agree up to all orders. In mathematics this conjecture has interesting applications in understanding the basic structure of link invariants and three manifold invariants, as well as the integrality structure of open Gromov-Witten invariants. Recently, X.S. Lin and H. Zheng [14] verified LMOV conjecture in several lower degree cases for some torus links.

In this paper, we describe an outline of the proof of Labastida-Mariño-Ooguri-Vafa conjecture for any link. The details of the proofs are given in [17.

\section{The Labastida-Mariño-Ooguri-Vafa conjecture}

2.1. Quantum group invariants of links. Let $\mathcal{L}$ be a link with $L$ components $\mathcal{K}_{\alpha}$, $\alpha=1, \ldots, L$, represented by the closure of an element of braid group $\mathcal{B}_{m}$. We associate to each $\mathcal{K}_{\alpha}$ an irreducible representation $R_{\alpha}$ of quantized universal enveloping algebra $U_{q}(\mathfrak{s l}(N, \mathbb{C}))$, labeled by its highest weight $\Lambda_{\alpha}$. Denote the corresponding module by $V_{\Lambda_{\alpha}}$. The $j$-th strand in the braid will be associated with the irreducible module $V_{j}=V_{\Lambda_{\alpha}}$, if this strand belongs to the component $\mathcal{K}_{\alpha}$. The braiding is defined through the following universal R-matrix of $U_{q}\left(\mathfrak{s l}_{N}\right)$

$$
\mathcal{R}=q^{\frac{1}{2} \sum_{i, j} C_{i j}^{-1} H_{i} \otimes H_{j}} \prod_{\text {positive root } \beta} \exp _{q}\left[\left(1-q^{-1}\right) E_{\beta} \otimes F_{\beta}\right] .
$$

Here $\left\{H_{i}, E_{i}, F_{i}\right\}$ are the generators of $U_{q}\left(\mathfrak{s l}_{N}\right),\left(C_{i j}\right)$ is the Cartan matrix and

$$
\exp _{q}(x)=\sum_{k=0}^{\infty} q^{\frac{1}{4} k(k+1)} \frac{x^{k}}{\{k\}_{q} !},
$$

where

$$
\{k\}_{q}=\frac{q^{-k / 2}-q^{k / 2}}{q^{-1 / 2}-q^{1 / 2}}, \quad\{k\}_{q} !=\prod_{j=1}^{k}\{j\}_{q} .
$$

Define braiding by $\check{\mathcal{R}}=P_{12} \mathcal{R}$, where $P_{12}(v \otimes w)=w \otimes v$.

\footnotetext{
${ }^{1}$ Later, we simply write $U_{q}\left(\mathfrak{s l}_{N}\right)$.
} 
Now for a given link $\mathcal{L}$ of $L$ components, one chooses a closed braid representative in braid group $\mathcal{B}_{m}$ whose closure is $\mathcal{L}$. In the case of no confusion, we also use $\mathcal{L}$ to refer its braid representative in $\mathcal{B}_{m}$. We will assign each crossing by the braiding as follows. Let $U, V$ be two $U_{q}\left(\mathfrak{s l}_{N}\right)$-modules labeling two outgoing strands of the crossing, the braiding $\check{R}_{U, V}$ (resp. $\check{R}_{V, U}^{-1}$ ) is assigned as in Figure 1 .
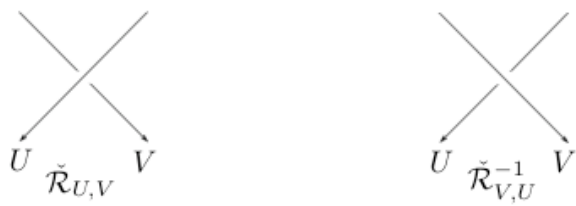

FiguRe 1. Assign crossing by $\check{\mathcal{R}}$.

The above assignment will give a representation of $\mathcal{B}_{m}$ on $U_{q}(\mathfrak{g})$-module $V_{1} \otimes \cdots \otimes V_{m}$. Namely, for any generator, $\sigma_{i} \in \mathcal{B}_{m}$, defin $\AA^{2}$

$$
h\left(\sigma_{i}\right)=\operatorname{id}_{V_{1}} \otimes \cdots \otimes \check{\mathcal{R}}_{V_{i}, V_{i+1}} \otimes \cdots \otimes i d_{V_{N}} .
$$

Therefore, any link $\mathcal{L}$ will provide an isomorphism

$$
h(\mathcal{L}) \in \operatorname{End}_{U_{q}\left(\mathfrak{s l}_{N}\right)}\left(V_{1} \otimes \cdots \otimes V_{m}\right) .
$$

Let $K_{2 \rho}$ be the enhancement of $\check{\mathcal{R}}$ in the sense of [24, where $\rho$ is the half-sum of all positive roots of $\mathfrak{s l}_{N}$. The irreducible representation $R_{\alpha}$ is labeled by the corresponding partition $A^{\alpha}$.

Definition 2.1. Given $L$ labeling partitions $A^{1}, \ldots, A^{L}$, the quantum group invariant of $\mathcal{L}$ is defined as follows:

$$
W_{\left(A^{1}, \ldots, A^{L}\right)}(\mathcal{L})=q^{d(\mathcal{L})} \operatorname{tr}_{V_{1} \otimes \cdots \otimes V_{m}}\left(K_{2 \rho} \circ h(\mathcal{L})\right),
$$

where

$$
d(\mathcal{L})=-\frac{1}{2} \sum_{\alpha=1}^{L} \omega\left(\mathcal{K}_{\alpha}\right)\left(\Lambda_{\alpha}, \Lambda_{\alpha}+2 \rho\right)+\frac{1}{N} \sum_{\alpha<\beta} \operatorname{lk}\left(\mathcal{K}_{\alpha}, \mathcal{K}_{\beta}\right)\left|A^{\alpha}\right| \cdot\left|A^{\beta}\right|,
$$

and $\operatorname{lk}\left(\mathcal{K}_{\alpha}, \mathcal{K}_{\beta}\right)$ is the linking number of components $\mathcal{K}_{\alpha}$ and $\mathcal{K}_{\beta}$. A substitution of $t=q^{N}$ is used to give a two-variable framing independent link invariant.

2.2. Labastida-Mariño-Ooguri-Vafa conjecture. Let $\mathcal{L}$ be a link with $L$ components and $\mathcal{P}$ be the set of all partitions. The Chern-Simons partition function of $\mathcal{L}$ is a generating function of quantum group invariants of links given by

$$
Z_{\mathrm{CS}}(\mathcal{L} ; q, t)=\sum_{\vec{A} \in \mathcal{P}^{L}} W_{\vec{A}}(\mathcal{L} ; q, t) \prod_{\alpha=1}^{L} s_{A^{\alpha}}\left(x^{\alpha}\right)
$$

for any arbitrarily chosen sequence of variables

$$
x^{\alpha}=\left(x_{1}^{\alpha}, x_{2}^{\alpha}, \ldots,\right) .
$$

In (2.1), $\vec{A}=\left(A^{1}, \ldots, A^{L}\right) \in \mathcal{P}^{L}$ and $s_{A^{\alpha}}\left(x^{\alpha}\right)$ is the Schur function.

\footnotetext{
${ }^{2}$ In the case of $\sigma_{i}^{-1}$, use $\check{\mathcal{R}}_{V_{i+1}, V_{i}}^{-1}$ instead.
} 
Free energy is defined to be

$$
F=\log Z_{\mathrm{CS}}
$$

Use plethystic exponential, one can obtain

$$
F=\sum_{d=1}^{\infty} \sum_{\vec{A} \neq 0} \frac{1}{d} f_{\vec{A}}\left(q^{d}, t^{d}\right) \prod_{\alpha=1}^{L} s_{A^{\alpha}}\left(\left(x^{\alpha}\right)^{d}\right)
$$

where

$$
\left(x^{\alpha}\right)^{d}=\left(\left(x_{1}^{\alpha}\right)^{d},\left(x_{2}^{\alpha}\right)^{d}, \ldots\right) .
$$

Based on the duality between Chern-Simons gauge theory and topological string theory, Labastida, Mariño, Ooguri, Vafa conjectured that $f_{\vec{A}}$ have the following highly nontrivial structures.

For any $A, B \in \mathcal{P}$, define the following function

$$
M_{A B}(q)=\sum_{\mu} \frac{\chi_{A}\left(C_{\mu}\right) \chi_{B}\left(C_{\mu}\right)}{\mathfrak{z}_{\mu}} \prod_{j=1}^{\ell(\mu)}\left(q^{-\mu_{j} / 2}-q^{\mu_{j} / 2}\right) .
$$

Conjecture (LMOV). For any $\vec{A} \in \mathcal{P}^{L}$,

(i). there exist $P_{\vec{B}}(q, t)$ for $\vec{B} \in \mathcal{P}^{L}$, such that

$$
f_{\vec{A}}(q, t)=\sum_{\left|B^{\alpha}\right|=\left|A^{\alpha}\right|} P_{\vec{B}}(q, t) \prod_{\alpha=1}^{L} M_{A^{\alpha} B^{\alpha}}(q) .
$$

Furthermore, $P_{\vec{B}}(q, t)$ has the following expansion

$$
P_{\vec{B}}(q, t)=\sum_{g=0}^{\infty} \sum_{Q \in \mathbb{Z} / 2} N_{\vec{B} ; g, Q}\left(q^{-1 / 2}-q^{1 / 2}\right)^{2 g-2} t^{Q} .
$$

(ii). $N_{\vec{B} ; g, Q}$ are integers.

2.3. Related notations. For a given $\operatorname{link} \mathcal{L}$ of $L$ components, we will fix the following notations in this paper. Given $\lambda \in \mathcal{P}, \vec{A}=\left(A^{1}, \ldots, A^{L}\right), \vec{\mu}=\left(\mu^{1}, \ldots, \mu^{L}\right) \in \mathcal{P}^{L}$. Let $x=\left(x^{1}, \ldots, x^{L}\right)$ where $x^{\alpha}$ is a set of variables

$$
x^{\alpha}=\left(x_{1}^{\alpha}, x_{2}^{\alpha}, \cdots\right) .
$$

The following notations will be used throughout the paper.

$$
\begin{array}{lll}
{[n]_{q}=q^{-\frac{n}{2}}-q^{\frac{n}{2}},} & {[\lambda]_{q}=\prod_{j=1}^{\ell(\lambda)}\left[\lambda_{j}\right]_{q},} & \mathfrak{z} \vec{\mu}=\prod_{\alpha=1}^{L} \mathfrak{z} \mu^{\alpha}, \\
|\vec{A}|=\left(\left|A^{1}\right|, \ldots,\left|A^{L}\right|\right), & \|\vec{A}\|=\sum_{\alpha=1}^{L}\left|A^{\alpha}\right|, & \ell(\vec{\mu})=\sum_{\alpha=1}^{L} \ell\left(\mu^{\alpha}\right), \\
\vec{A}^{t}=\left(\left(A^{1}\right)^{t}, \ldots,\left(A^{L}\right)^{t}\right), & \chi_{\vec{A}}(\vec{\mu})=\prod_{\alpha=1}^{L} \chi_{A^{\alpha}}\left(C_{\mu^{\alpha}}\right), & s_{\vec{A}}(x)=\prod_{\alpha=1}^{L} s_{A^{\alpha}}\left(x^{\alpha}\right) .
\end{array}
$$


One can define an order on $\mathcal{P}^{L}$ lexicographically. Therefore, one can generalize the concept of partition from the set of all non-negative integers to $\mathcal{P}^{L}$. We denote by $\mathcal{P}\left(\mathcal{P}^{L}\right)$ the set of all partitions on $\mathcal{P}^{L}$. Given $\Lambda \in \mathcal{P}\left(\mathcal{P}^{L}\right)$, the following quantity

$$
\theta_{\Lambda}=\frac{(-1)^{\ell(\Lambda)-1}(\ell(\Lambda)-1) !}{|\operatorname{Aut} \Lambda|}
$$

plays an important role in the relationship of topological string partition function and free energy.

Rewrite free energy as

$$
F=\log Z=\sum_{\vec{\mu} \neq 0} F_{\vec{\mu}} p_{\vec{\mu}}(x) .
$$

Here in the similar usage of notation,

$$
p_{\vec{\mu}}(x)=\prod_{\alpha=1}^{L} p_{\mu^{\alpha}}\left(x^{\alpha}\right) .
$$

We also rewrite Chern-Simons partition function as

$$
Z_{\mathrm{CS}}(\mathcal{L})=1+\sum_{\vec{\mu} \neq 0} Z_{\vec{\mu}} p_{\vec{\mu}}(x) .
$$

\section{Sketch of the proof}

In this section we will give an outline of the proof of Labastida-Mariño-Ooguri-Vafa conjecture ${ }^{3}$ which contains two parts in correspondence of (i) and (ii) in the description of LMOV conjecture: the existence and integrality.

3.1. Existence of the algebraic structure. The existence of the algebraic structure 2.5 includes the following two steps:

- The symmetry of $q$ and $q^{-1}$ in $P_{\vec{B}}(q, t)$.

- The pole structure of $P_{\vec{B}}$.

The symmetry of $q$ and $q^{-1}$ in $P_{\vec{B}}(q, t)$ can be obtained from the lemma.

Lemma 3.1. $W_{\vec{A}^{t}}(q, t)=(-1)^{\|\vec{A}\|} W_{\vec{A}}\left(q^{-1}, t\right)$.

To prove the existence of the pole structure, we will consider the following framed generating series. Substitute

$$
W_{\vec{A}}(\mathcal{L} ; q, t, \tau)=W_{\vec{A}}(\mathcal{L} ; q, t) \cdot q^{\sum_{\alpha=1}^{L} \kappa_{A} \alpha \tau / 2}
$$

in the Chern-Simons partition function, we have the following framed partition function

$$
Z(\mathcal{L} ; q, t, \tau)=1+\sum_{\vec{A} \neq 0} W_{\vec{A}}(\mathcal{L} ; q, t, \tau) \cdot s_{\vec{A}}(x) .
$$

Similarly, framed free energy

$$
F(\mathcal{L} ; q, t, \tau)=\log Z(\mathcal{L} ; q, t, \tau) .
$$

\footnotetext{
${ }^{3}$ Briefly, we call it LMOV conjecture.
} 
It satisfies the following cut-and-join equation

$$
\frac{\partial F(\mathcal{L} ; q, t, \tau)}{\partial \tau}=\frac{u}{2} \sum_{\alpha=1}^{L} \sum_{i, j \geq 1}\left(i j p_{i+j}^{\alpha} \frac{\partial^{2} F}{\partial p_{i}^{\alpha} \partial p_{j}^{\alpha}}+(i+j) p_{i}^{\alpha} p_{j}^{\alpha} \frac{\partial F}{\partial p_{i+j}^{\alpha}}+i j p_{i+j}^{\alpha} \frac{\partial F}{\partial p_{i}^{\alpha}} \frac{\partial F}{\partial p_{j}^{\alpha}}\right)
$$

Restrict the equation to $\vec{\mu}$, we have

$$
\frac{\partial F_{\vec{\mu}}}{\partial \tau}=\frac{u}{2}\left(\sum_{|\vec{\nu}|=|\vec{\mu}|, \ell(\vec{\nu})=\ell(\vec{\mu}) \pm 1} \alpha_{\vec{\mu} \vec{\nu}} F_{\vec{\nu}}+\text { nonlinear terms }\right)
$$

where $\alpha_{\vec{\mu} \vec{\nu}}$ is some constant.

Denote by $\operatorname{deg}_{u}$ the lowest degree of $u$ in a Laurent polynomial of $u$. The pole structure of $P_{\vec{B}}$ follows from the following degree lemma:

Lemma 3.2. $\operatorname{deg}_{u} F_{\vec{\mu}} \geq \ell(\vec{\mu})-2$.

Let $\bigcirc$ be the unknot. Given any $\vec{A}=\left(A^{1}, \ldots, A^{L}\right) \in \mathcal{P}^{L}$, we will obtain the following limit behavior of quantum group invariants of links at $q \rightarrow 1$.

$$
\lim _{q \rightarrow 1} \frac{W_{\vec{A}}(\mathcal{L} ; q, t)}{W_{\vec{A}}\left(\bigcirc^{\otimes L} ; q, t\right)}=\prod_{\alpha=1}^{L} \xi_{\mathcal{K}_{\alpha}}(t)^{d_{\alpha}}
$$

where $\left|A^{\alpha}\right|=d_{\alpha}, \mathcal{K}_{\alpha}$ is the $\alpha$-th component of $\mathcal{L}$, and $\xi_{\mathcal{K}_{\alpha}}(t), \alpha=1, \ldots, L$, are independent of $\vec{A}$.

The following cut-and-join analysis will give the desired results.

If $\|\vec{\mu}\|=1$, it can be verified through the degree of $u$ in HOMFLY polynomial.

If $\|\vec{\mu}\|>1$, by 3.2 We can prove that

$$
\operatorname{deg}_{u}\left(\frac{\partial F_{\vec{\mu}}}{\partial \tau}\right)=\operatorname{deg}_{u} F_{\vec{\mu}}
$$

Suppose Lemma 3.2 holds for any $\|\vec{\nu}\|<d$. Now, consider

$$
S=\{\vec{\nu}:|\vec{\nu}|=\vec{d}\}
$$

where $\vec{d}=\left(d^{1}, \ldots, d^{L}\right)$ and $\sum_{\alpha=1}^{L} d^{\alpha}=d$. If $\vec{\mu} \in S$, one important fact is that the degree of $u$ in the nonlinear terms in 3.1 is no less than $\ell(\vec{\mu})-3$. We will prove Lemma 3.2 holds for $\forall \vec{\mu} \in S$ by contradiction.

Denote by

$$
S_{r}=\{\vec{\nu}: \vec{\nu} \in S \text { and } \ell(\vec{\nu})=r\} \subset S
$$

and

$$
D_{r}=\min \left\{\operatorname{deg}_{u} F_{\vec{\mu}}: \vec{\mu} \in S_{r}\right\} .
$$

Assume $k$ is the smallest integer such that there exists a $\vec{\nu} \in S_{k}$ satisfying

$$
\operatorname{deg}_{u} F_{\vec{\nu}}<\ell(\vec{\nu})-2=k-2 .
$$

Choose $\vec{\mu} \in S_{k}$ such that $\operatorname{deg}_{u} F_{\vec{\mu}}=D_{k}$. Rewrite 3.1 into three parts

$$
\frac{\partial F_{\vec{\mu}}}{\partial \tau}=\frac{u}{2} \sum_{|\vec{\nu}|=|\vec{\mu}|, \ell(\vec{\nu})=\ell(\vec{\mu})-1} \alpha_{\vec{\mu} \vec{\nu}} F_{\vec{\nu}}+\frac{u}{2} \sum_{|\vec{\nu}|=|\vec{\mu}|, \ell(\vec{\nu})=\ell(\vec{\mu})+1} \alpha_{\vec{\mu} \vec{\nu}} F_{\vec{\nu}}+*
$$

where $*$ represents the nonlinear part and $\operatorname{deg}_{u} *=k-2$. Here in the equation, $\vec{\nu}$ runs in $S$. 
However,

$$
\operatorname{deg}_{u} \frac{\partial F_{\vec{\mu}}}{\partial \tau}=\operatorname{deg}_{u} F_{\vec{\mu}}=D_{k}<k-2 .
$$

$D_{k-1} \geq(k-1)-2$, so the first part of the sums in the r.h.s of $(3.3)$ is of the lowest order of $u$ at least $k-2$ and the lowest order of $u$ in $\frac{\partial F_{\vec{\mu}}}{\partial \tau}$ must come from the summation over $\ell(\vec{\nu})=\ell(\vec{\mu})+1$. Then we have

$$
1+D_{k+1} \leq D_{k}
$$

and

$$
D_{k+1} \leq D_{k}-1<k-2-1<(k+1)-2 .
$$

Choose $\vec{\xi} \in S_{k+1}$ such that $\operatorname{deg}_{u} F_{\vec{\xi}}=D_{K+1}$, run the similar analysis as above. Since (3.4, one will get

$$
1+D_{k+2} \leq D_{k+1}
$$

Thus we obtain

$$
D_{k}>D_{k+1}>D_{k+2}>\ldots D_{d-1}>D_{d} .
$$

Remember that there is still one last equation we have not used yet. Consider the cut-and-join equation for the following partition

$$
\vec{\eta}=\left(\left(1^{d_{1}}\right), \ldots,\left(1^{d_{L}}\right)\right) \in S_{d}
$$

Note that this partition has the longest length in $S$, there will be only the first summand in (3.3) left. In particular, no non-linear terms will appear in the equation. Therefore,

$$
\frac{\partial F_{\vec{\eta}}}{\partial \tau}=\frac{u}{2} \sum_{\ell(\vec{\nu})=d-1} \alpha_{\vec{\eta} \vec{\nu}} F_{\vec{\nu}}
$$

This implies

$$
D_{d} \geq D_{d-1}+1
$$

which contradicts with $D_{d-1}>D_{d}$. This gives a proof of the existence of $(2.5)$.

Define

$$
\widetilde{F}_{\vec{\mu}}=\frac{F_{\vec{\mu}}}{\phi_{\vec{\mu}}(q)}, \quad \widetilde{Z}_{\vec{\mu}}=\frac{Z_{\vec{\mu}}}{\phi_{\vec{\mu}}(q)} .
$$

Lemma 3.2 directly implies the following:

Corollary 3.3. $\widetilde{F}$ are of the following form:

$$
\widetilde{F}_{\vec{\mu}}(q, t)=\sum_{\text {finitely many } n_{\alpha}} \frac{a_{\alpha}(t)}{\left[n_{\alpha}\right]^{2}}+\text { polynomial. }
$$

Let $\mathcal{L}$ be the closure of a zero writhe braid $\beta$. Cabling the $\alpha$-th component of $\beta$ into $\ell\left(\mu^{\alpha}\right)$ parallel ones and coloring them with partitions $\left(\mu_{1}^{\alpha}\right), \ldots,\left(\mu_{\ell\left(\mu^{\alpha}\right)}^{\alpha}\right)$, we close the corresponding braid and denote it to be $\mathcal{L}_{\vec{\mu}}$.

Let $\mu^{i}=\left(\mu_{1}^{i}, \cdots, \mu_{\ell_{i}}^{i}\right)$. We use symbol

$$
\hat{Z}_{\vec{\mu}}=Z_{\vec{\mu}} \cdot \mathfrak{z}_{\vec{\mu}} ; \quad \hat{F}_{\vec{\mu}}=F_{\vec{\mu}} \cdot \mathfrak{z}_{\vec{\mu}} \cdot
$$


Notice the following fact from the definition of quantum group invariants:

$$
\begin{aligned}
& \hat{Z}_{\left(\mu^{1}, \cdots, \mu^{L}\right)}(\mathcal{L})=\hat{Z}_{\left(\mu_{1}^{1}\right), \cdots,\left(\mu_{\ell_{1}}^{1}\right), \cdots,\left(\mu_{1}^{L}\right), \cdots,\left(\mu_{\ell_{L}}^{L}\right)}\left(\mathcal{L}_{\vec{\mu}}\right), \\
& \hat{F}_{\left(\mu^{1}, \cdots, \mu^{L}\right)}(\mathcal{L})=\hat{F}_{\left(\mu_{1}^{1}\right), \cdots,\left(\mu_{\ell_{1}}^{1}\right), \cdots,\left(\mu_{1}^{L}\right), \cdots,\left(\mu_{\ell_{L}}^{L}\right)}\left(\mathcal{L}_{\vec{\mu}}\right) .
\end{aligned}
$$

Consider $\delta_{n}=\sigma_{1} \cdots \sigma_{n-1}$. Let $\mathfrak{S}_{A}$ be the minimal projection from $\mathcal{H}_{n} \rightarrow \mathcal{H}_{A}$, and

$$
\mathfrak{P}_{\mu}=\sum_{A} \chi_{A}\left(C_{\mu}\right) \mathfrak{S}_{A}
$$

We will apply a lemma of Aiston-Morton [2] in the following computation:

$$
\delta_{n}^{n} \mathfrak{S}_{A}=q^{\frac{1}{2} \kappa_{A}} \mathfrak{S}_{A} .
$$

Let

$$
\vec{d}=\left(\left(d_{1}\right), \ldots,\left(d_{L}\right)\right), \quad \frac{1}{\vec{d}}=\left(\frac{1}{d_{1}}, \ldots, \frac{1}{d_{L}}\right) .
$$

Due to the cabling formula to the length of partition (3.5) and (3.6), we can simply deal with all the color of one row without loss of generality. Take framing $\tau_{\alpha}=n_{\alpha}+\frac{1}{d_{\alpha}}$ and choose a braid group representative of $\mathcal{L}$ such that the writhe number of $\mathcal{L}_{\alpha}$ is $n_{\alpha}$. Denote by $\vec{\tau}=\left(\tau_{1}, \ldots, \tau_{L}\right)$,

$$
\begin{aligned}
\hat{Z}_{\vec{d}}(\mathcal{L} ; q, t ; \vec{\tau}) & =\sum_{\vec{A}} \chi_{\vec{A}}\left(C_{\vec{d}}\right) W_{\vec{A}}(\mathcal{L} ; q, t) q^{\frac{1}{2} \sum_{\alpha=1}^{L} \kappa_{A^{\alpha}}\left(n_{\alpha}+\frac{1}{d_{\alpha}}\right)} \\
& =t^{\frac{1}{2} \sum_{\alpha} d_{\alpha} n_{\alpha}} \operatorname{Tr}\left(\mathcal{L}_{\vec{d}} \sum_{\vec{A}} \chi_{\vec{A}}\left(C_{\vec{d}}\right) q^{\frac{1}{2} \sum_{\alpha} \kappa_{\alpha} \frac{1}{d_{\alpha}}} \bigotimes_{\alpha} \mathfrak{S}_{A^{\alpha}}\right) \\
& =t^{\frac{1}{2} \sum_{\alpha} d_{\alpha} n_{\alpha}} \operatorname{Tr}\left(\mathcal{L}_{\vec{d}} \sum_{\vec{A}} \chi_{\vec{A}}\left(C_{\vec{d}}\right)\left(\delta_{d_{1}} \otimes \cdots \otimes \delta_{d_{L}}\right) \bigotimes_{\alpha} \mathfrak{S}_{A^{\alpha}}\right) \\
& =t^{\frac{1}{2} \sum_{\alpha} d_{\alpha} n_{\alpha}} \operatorname{Tr}\left(\mathcal{L}_{\vec{d}} \cdot \otimes_{\alpha=1}^{L} \delta_{d_{\alpha}} \cdot \mathfrak{P}_{(1)}^{\left(d_{1}\right)} \otimes \cdots \otimes \mathfrak{P}_{(1)}^{\left(d_{L}\right)}\right) .
\end{aligned}
$$

Here, $\mathfrak{P}_{(1)}^{\left(d_{\alpha}\right)}$ means that in the projection, we use $q^{d_{\alpha}}, t^{d_{\alpha}}$ instead of using $q, t$. If we denote by

$$
\mathcal{L} * Q_{\vec{d}}=\mathcal{L}_{\vec{d}} \cdot \delta_{\vec{d}} \cdot \mathfrak{P}_{(1)}^{\left(d_{1}\right)} \otimes \cdots \otimes \mathfrak{P}_{(1)}^{\left(d_{L}\right)}
$$

we have

$$
\hat{Z}_{\vec{d}}\left(\mathcal{L} ; q, t ; \frac{1}{\vec{d}}\right)=\mathcal{H}\left(\mathcal{L} * Q_{\vec{d}}\right) .
$$

Here $\mathcal{H}$ is the homfly polynomial which is normalized as

$$
\mathcal{H}(\text { unknot })=\frac{t^{\frac{1}{2}}-t^{-\frac{1}{2}}}{q^{\frac{1}{2}}-q^{-\frac{1}{2}}}
$$

With the above normalization, for any given link $\mathcal{L}$, we have

$$
[1]^{L} \cdot \mathcal{H}(\mathcal{L}) \in \mathbb{Q}\left[[1]^{2}, t^{ \pm \frac{1}{2}}\right] .
$$

Substituting $q$ by $q^{d_{\alpha}}$ in the corresponding component, it lead to the following:

$$
\prod_{\alpha=1}^{L}\left[d_{\alpha}\right] \cdot \hat{Z}_{\vec{d}}(\mathcal{L} ; q, t ; \vec{\tau}) \in \mathbb{Q}\left[[1]^{2}, t^{ \pm \frac{1}{2}}\right] .
$$


On the other hand, given any frames $\vec{\omega}=\left(\omega_{1}, \ldots, \omega_{L}\right)$,

$$
\begin{aligned}
\hat{Z}_{\vec{\mu}}(\mathcal{L} ; q, t ; \vec{\omega}) & =\sum_{\vec{A}} \chi_{\vec{A}}\left(C_{\vec{\mu}}\right) W_{\vec{A}}(\mathcal{L} ; q, t ; \vec{\omega}) \\
& =\sum_{\vec{A}} \chi_{\vec{A}}\left(C_{\vec{\mu}}\right) \sum_{\vec{\nu}} \frac{\chi_{\vec{A}}\left(C_{\vec{\nu}}\right)}{\mathfrak{z}_{\vec{\nu}}} \hat{Z}_{\vec{\nu}}(\mathcal{L} ; q, t) q^{\frac{1}{2} \sum_{\alpha} \kappa_{A} \alpha \omega_{\alpha}} .
\end{aligned}
$$

Exchange the order of summation, we have the following convolution formula:

$$
\hat{Z}_{\vec{\mu}}(\mathcal{L} ; q, t ; \vec{\omega})=\sum_{\vec{\nu}} \frac{\hat{Z}_{\vec{\nu}}(\mathcal{L} ; q, t)}{\mathfrak{z} \vec{\nu}} \sum_{\vec{A}} \chi_{\vec{A}}\left(C_{\vec{\mu}}\right) \chi_{\vec{A}}\left(C_{\vec{\nu}}\right) q^{\frac{1}{2} \sum_{\alpha} \kappa_{A} \alpha \omega_{\alpha}} .
$$

This property holds for arbitrary choice of $n_{\alpha}, \alpha=1, \ldots, L$. The coefficients of possible other poles vanish for arbitrary integer $n_{\alpha} . q^{n_{\alpha}}$ is involved through certain polynomial relation, which implies the coefficients for other possible poles are simply zero. Therefore, (3.8) holds for any frame.

Now instead of canceling all the poles of $\hat{Z}(\mathcal{L})$ according to $(3.8)$, we consider $[c] \hat{Z}_{(c), \vec{d}}$, which is equivalent to consider $[c]^{2} \widetilde{Z}_{(c), \vec{d}}$. Let $\mathcal{K}$ be the knot labeled by $[c]$. Multiplying $\widetilde{Z}$ by $[c]^{2}$ cancels all the poles related to $\mathcal{K}$ according to $(3.7)$. Therefore, $[c]^{2} \widetilde{Z}_{(c), \vec{d}}$ has the same principal part as $\widetilde{Z}_{\vec{d}}$ except for multiplying by an element in $\mathbb{Q}\left[[1]^{2}, t^{ \pm \frac{1}{2}}\right]$. Note that

$$
\widetilde{Z}_{\vec{\mu}}=\sum_{\Lambda \vdash \vec{\mu}} \frac{\widetilde{F}_{\Lambda}}{\operatorname{Aut}|\Lambda|} .
$$

$[c]^{2}\left(\widetilde{Z}_{(c), \vec{d}}-\widetilde{F}_{(c), \vec{d}}\right)$ contains all the principal terms from $\mathcal{L}$ omitting $\mathcal{K}$. Therefore,

$$
[c]^{2} \widetilde{F}_{(c), \vec{d}} \in \mathbb{Q}\left[[1]^{2}, t^{ \pm \frac{1}{2}}\right] .
$$

In the above discussion, $\mathcal{K}$ can be chosen to be any component of $\mathcal{L}$. We thus proved the following proposition:

Proposition 3.4. Notations as above, we have:

$$
\begin{aligned}
\prod_{\alpha=1}^{L}\left[d_{\alpha}\right] \cdot \hat{Z}_{\vec{d}}(\mathcal{L} ; q, t) & \in \mathbb{Q}\left[[1]^{2}, t^{ \pm \frac{1}{2}}\right] ; \\
& {\left[d_{\alpha}\right]^{2} \widetilde{F}_{\vec{d}}(\mathcal{L} ; q, t) \in \mathbb{Q}\left[[1]^{2}, t^{ \pm \frac{1}{2}}\right], \forall \alpha . }
\end{aligned}
$$

Similarly, We can obtain

$$
\widetilde{F}_{\vec{d}}(\mathcal{L} ; q, t)=\frac{H_{\vec{d} / D_{\vec{d}}}\left(t^{D_{\vec{d}}}\right)}{\left[D_{\vec{d}}\right]^{2}}+\text { polynomial in }\left[D_{\vec{d}}\right]^{2} \text { and } t^{ \pm \frac{1}{2} D_{\vec{d}}} .
$$

Once again, due to arbitrary choice of $n_{\alpha}$, we know the above pole structure of $\widetilde{F}_{\vec{d}}$ holds for any frame. 
Proposition 3.5. Notations are as above. Assume $\mathcal{L}$ is labeled by the color $\vec{\mu}=$ $\left(\mu^{1}, \ldots, \mu^{L}\right)$. Denote by $D_{\vec{\mu}}$ is the greatest common divisor of $\left\{\mu_{1}^{1}, \ldots, \mu_{\ell\left(\mu^{1}\right)}^{1}, \ldots\right.$, $\left.\mu_{j}^{i}, \ldots, \mu_{\ell\left(\mu^{L}\right)}^{L}\right\} . \widetilde{F}_{\vec{\mu}}$ has the following structure:

$$
\widetilde{F}_{\vec{\mu}}(q, t)=\frac{H_{\vec{\mu} / D_{\vec{\mu}}}\left(t^{D_{\vec{\mu}}}\right)}{\left[D_{\vec{\mu}}\right]^{2}}+f(q, t),
$$

where $H_{\vec{\mu} / D_{\vec{\mu}}}(t)$ only depends on $\vec{\mu} / D_{\vec{\mu}}$ and $\mathcal{L}, f(q, t) \in \mathbb{Q}\left[[1]^{2}, t^{ \pm \frac{1}{2}}\right]$.

Remark 3.6. In Proposition 3.5, it is very interesting to interpret in topological string side that $H_{\vec{\mu} / D_{\vec{\mu}}}(t)$ only depends on $\vec{\mu} / D_{\vec{\mu}}$ and $\mathcal{L}$. The principal term is generated due to summation of counting rational curves and independent choice of $k$ in the labeling color $k \cdot \vec{\mu} / D_{\vec{\mu}}$. This phenomenon simply tells us that contributions of counting rational curves in the labeling color $k \cdot \vec{\mu} / D_{\vec{\mu}}$ are through multiple cover contributions of $\vec{\mu} / D_{\vec{\mu}}$.

3.2. Integrality. By the definition of $P_{\vec{B}}(q, t)$, comparing with Proposition 3.5 , we have the following computation:

$$
\begin{aligned}
P_{\vec{B}}(q, t) & =\sum_{\vec{\mu}} \frac{\chi_{\vec{B}}(\vec{\mu})}{\phi_{\vec{\mu}}(q)} \sum_{d \mid \vec{\mu}} \frac{\pi(d)}{d} F_{\vec{\mu} / d}\left(q^{d}, t^{d}\right) \\
& =\sum_{\vec{\mu}} \chi_{\vec{B}}(\vec{\mu}) \sum_{d \mid \vec{\mu}} \frac{\mu(d)}{d} \widetilde{F}_{\vec{\mu} / d}\left(q^{d}, t^{d}\right) \\
& =\sum_{\vec{\mu}} \chi_{\vec{B}}(\vec{\mu}) \sum_{d \mid D_{\vec{\mu}}} \frac{\mu(d)}{d} \frac{H_{\vec{\mu} / D_{\vec{\mu}}}\left(t^{D_{\vec{\mu}}}\right)}{\left[D_{\vec{\mu}}\right]^{2}}+\text { polynomial } \\
& =\sum_{\vec{\mu}} \chi_{\vec{B}}(\vec{\mu}) \delta_{1, D_{\vec{\mu}}} \frac{H_{\vec{\mu} / D_{\vec{\mu}}}\left(t^{D_{\vec{\mu}}}\right)}{\left[D_{\vec{\mu}}\right]^{2}}+\text { polynomial },
\end{aligned}
$$

where $\delta_{1, n}$ equals 1 if $n=1$ and 0 otherwise. It implies that $P_{\vec{B}}$ is a rational function which only has pole at $q=1$. In the above computation, we used a fact of Möbius inversion,

$$
\sum_{d \mid n} \frac{\mu(d)}{d}=\delta_{1, n}
$$

Therefore, for each $\vec{B}$,

$$
\sum_{g=0}^{\infty} \sum_{Q \in \mathbb{Z} / 2} N_{\vec{B} ; g, Q}\left(q^{-1 / 2}-q^{1 / 2}\right)^{2 g} t^{Q} \in \mathbb{Q}\left[\left(q^{-1 / 2}-q^{1 / 2}\right)^{2}, t^{ \pm \frac{1}{2}}\right] .
$$

The integrality of $N_{\vec{B} ; g, Q}$ can be derived from the following theorem:

Theorem 1. We have

$$
\sum_{g=0}^{\infty} \sum_{Q \in \mathbb{Z}} N_{\vec{B} ; g, Q}\left(q^{-1 / 2}-q^{1 / 2}\right)^{2 g} t^{Q} \in \mathbb{Z}\left[\left(q^{-1 / 2}-q^{1 / 2}\right)^{2}, t^{ \pm 1 / 2}\right] .
$$


Remark 3.7. The above theorem also implies the refined integral invariants $N_{\vec{B} ; g, Q}$ vanish at large genera (also for large $Q$ ).

Define $y=\left(y^{1}, y^{2}, \ldots, y^{L}\right)$ where

$$
y^{\alpha}=\left(y_{1}^{\alpha}, y_{2}^{\alpha}, \ldots\right)
$$

is a set of arbitrarily chosen variables. Denote by $\Omega(y)$ the set of all symmetric function in $\left(y^{1}, y^{2}, \ldots, y^{L}\right)$ with integral coefficients.

We start by defining the following special ring which characterizing the algebraic structure of Chern-Simons partition function.

$$
\mathfrak{R}(y ; q, t)=\left\{\frac{a(y ; q, t)}{b(q)}: a(y ; q, t) \in \Omega(y)\left[[1]_{q}^{2}, t^{ \pm 1 / 2}\right], b(q)=\prod_{n_{k}}\left[n_{k}\right]_{q}^{2} \in \mathbb{Z}\left[[1]_{q}^{2}\right]\right\} .
$$

Given $\frac{f(y ; q, t)}{b(q)} \in \mathfrak{R}(y ; q, t)$, if $f(y ; q, t)$ is a primitive polynomial in terms of $q^{ \pm 1 / 2}$, $t^{ \pm 1 / 2}$ and Schur functions of $y$, we call $\frac{f(y ; q, t)}{b(q)}$ is primitive.

Given any $\frac{r}{s} h(y ; q, t)$ where $h(y ; q, t) \in \mathfrak{R}(y ; q, t)$ is primitive, define

$$
\operatorname{Ord}_{p}\left(\frac{r}{s} h(y ; q, t)\right)=\operatorname{Ord}_{p}\left(\frac{r}{s}\right)
$$

for any prime number $p$.

We will consider the following generating series

$$
T_{\vec{d}}=\sum_{|\vec{B}|=\vec{d}} s_{\vec{B}}(y) P_{\vec{B}}(q, t)
$$

After some calculations, we have

$$
T_{\vec{d}}=q^{|\vec{d}|} \sum_{k \mid \vec{d}} \frac{\mu(k)}{k} \sum_{\mathfrak{A} \in \mathcal{P}\left(\mathcal{P}^{n}\right),\|\mathfrak{A}\|=\vec{d} / k} \theta_{\mathfrak{A}} \prod_{j=1}^{\ell(\mathfrak{A})} W_{\mathfrak{A}_{j}}\left(q^{k}, t^{k}\right) s_{\mathfrak{A}_{j}}\left((z)^{k}\right)
$$

Proposition 3.8. $T_{\vec{d}}(y ; q, t) \in \mathfrak{R}(y ; v, t)$.

Proposition 3.8 implies $q=0$ is a pole of $T_{\vec{d}}$. Since $T_{\vec{d}}$ can be written as

$$
T_{\vec{d}}=\sum_{g=0}^{\infty} \sum_{Q \in \mathbb{Z} / 2}\left(\sum_{|\vec{B}|=\vec{d}} N_{\vec{B} ; g, Q} s_{\vec{B}}(y)\right)\left(q^{-1 / 2}-q^{1 / 2}\right)^{2 g-2} t^{Q}
$$

by the existence of 2.5). If there are infinitely many $N_{\vec{B} ; g, Q}$ nonzero, $q=0$ is then an essential singularity point of $T_{\vec{d}}$, which is a contradiction. Therefore, for each $\vec{B}$,

$$
\sum_{g=0}^{\infty} \sum_{Q \in \mathbb{Z} / 2} N_{\vec{B} ; g, Q}\left(q^{-1 / 2}-q^{1 / 2}\right)^{2 g} t^{Q} \in \mathbb{Q}\left[\left(q^{-1 / 2}-q^{1 / 2}\right)^{2}, t^{ \pm \frac{1}{2}}\right] .
$$

On the other hand, by Proposition $3.8 . T_{\vec{d}} \in \mathfrak{R}(y ; v, t)$ and $\operatorname{Ord}_{p} T_{\vec{d}} \geq 0$ for any prime number $p$. We have

$$
\sum_{|\vec{B}|=\vec{d}} N_{\vec{B} ; g, Q} s_{\vec{B}}(y) \in \Omega(y),
$$

which implies $N_{\vec{B} ; g, Q} \in \mathbb{Z}$. 
Combining the above discussions, we have

$$
\sum_{g=0}^{\infty} \sum_{Q \in \mathbb{Z} / 2} N_{\vec{B} ; g, Q}\left(q^{-1 / 2}-q^{1 / 2}\right)^{2 g} t^{Q} \in \mathbb{Z}\left[\left(q^{-1 / 2}-q^{1 / 2}\right)^{2}, t^{ \pm 1 / 2}\right] .
$$

To prove Proposition 3.8, we combine the study of multi-cover contribution and $p$-adic argument. For any give prime number $p$, the following observation is important for the $p$-adic argument

$$
\left\{\mathfrak{B}: \sum_{j=1}^{\ell(\mathfrak{B})}\left|\mathfrak{B}_{j}\right|=p \vec{d} \text { and } \operatorname{Ord}_{p}\left(\theta_{\mathfrak{B}}\right)<0\right\}=\left\{\mathfrak{A}^{(p)}: \sum_{j=1}^{\ell(\mathfrak{A})}\left|\mathfrak{A}_{j}\right|=\vec{d}\right\} .
$$

Matching the following terms, finally we have

$$
\operatorname{Ord}_{p}\left(\theta_{\mathfrak{A}^{(p)}} W_{\mathfrak{A}^{(p)}}(q, t) s_{\mathfrak{A}^{(p)}}(z)-\frac{1}{p} \theta_{\mathfrak{A}} W_{\mathfrak{A}}\left(q^{p}, t^{p}\right) s_{\mathfrak{A}}\left(z^{p}\right)\right) \geq 0 .
$$

Let

$$
\Phi_{\vec{d}}(y ; q, t)=\sum_{\mathfrak{A} \in \mathcal{P}\left(\mathcal{P}^{n}\right),\|\mathfrak{A}\|=\vec{d}} \theta_{\mathfrak{A}} W_{\mathfrak{A}}(q, t) s_{\mathfrak{A}}(z) .
$$

The following inequality can be obtained from 3.15$)$ :

$$
\operatorname{Ord}_{p}\left(\Phi_{p \vec{d}}(y ; q, t)-\frac{1}{p} \Phi_{\vec{d}}\left(y^{p} ; q^{p}, t^{p}\right)\right) \geq 0
$$

Therefore,

$$
\begin{aligned}
T_{\vec{d}} & =q^{|\vec{d}|} \sum_{k \mid \vec{d}} \frac{\mu(k)}{k} \sum_{\mathfrak{A} \in \mathcal{P}\left(\mathcal{P}^{L}\right),\|\mathfrak{A}\|=\vec{d} / k} \theta_{\mathfrak{A}} W_{\mathfrak{A}}\left(q^{k}, t^{k}\right) s_{\mathfrak{A}}\left(z^{k}\right) \\
& =q^{|\vec{d}|} \sum_{k \mid \vec{d}, p \nmid k} \frac{\mu(k)}{k}\left(\Phi_{\vec{d} / k}\left(y^{k} ; q^{k}, t^{k}\right)-\frac{1}{p} \Phi_{\vec{d} /(p k)}\left(y^{p k} ; q^{p k}, t^{p k}\right)\right) .
\end{aligned}
$$

This implies

$$
\operatorname{Ord}_{p} T_{\vec{d}} \geq 0
$$

Combining with 2.5), we thus prove Proposition 3.8

\section{Concluding remarks}

In this section, we briefly discuss some interesting problems related to string duality which may be approached through the techniques developed in this paper.

Let $\mathbf{p}=\left(\mathbf{p}^{1}, \ldots, \mathbf{p}^{L}\right)$, where $\mathbf{p}^{\alpha}=\left(p_{1}^{\alpha}, p_{2}^{\alpha}, \ldots,\right)$. Defined the following generating series of open Gromov-Witten invariants

$$
F_{g, \vec{\mu}}(t, \tau)=\sum_{\beta} K_{g, \vec{\mu}}^{\beta}(\tau) e^{\int_{\beta} \omega}
$$

where $\omega$ is the Kähler class of the resolved conifold, $\tau$ is the framing parameter and

$$
t=e^{\int_{\mathbb{P} 1} \omega}, \quad \text { and } \quad e^{\int_{\beta} \omega}=t^{Q} .
$$


Consider the following generating function

$$
F(\mathbf{p} ; u, t ; \tau)=\sum_{g=0}^{\infty} \sum_{\vec{\mu}} u^{2 g-2+\ell(\vec{\mu})} F_{g, \vec{\mu}}(t ; \tau) \prod_{\alpha=1}^{L} p_{\mu^{\alpha}}^{\alpha} .
$$

It satisfies the log cut-and-join equation

$$
\frac{\partial F(\mathbf{p} ; u, t ; \tau)}{\partial \tau}=\frac{u}{2} \sum_{\alpha=1}^{L} \mathfrak{L}_{\alpha} F(\mathbf{p} ; u, t ; \tau) .
$$

Therefore, duality between Chern-Simons theory and open Gromov-Witten theory reduces to verifying the uniqueness of the solution of cut-and-join equation.

Cut-and-join equation for Gromov-Witten side comes from the degeneracy and gluing procedure while uniqueness of cut-and-join system should in principle be obtained from the verification at some initial value. However, it seems very difficult to find a suitable initial value. A new hope might be found in our development of cut-and-join analysis. In the log cut-and-join equation the non-linear terms reveals the important recursion structure. For the uniqueness of cut-and-join equation, it will appear as the vanishing of all non-linear terms. We will put this in our future research.

Our proof of the LMOV conjecture has shed new light on the famous volume conjecture. See for example the discussions in [6]. The cut-and-join analysis we developed in this paper combined with rank-level duality in Chern-Simons theory seems to provide a new way to prove the existence of the limits of quantum invariants.

There are also other open problems related to LMOV conjecture. For example, quantum group invariants satisfy skein relation which must have some implications on topological string side as mentioned in [10]. One could also rephrase a lot of unanswered questions in knot theory in terms of open Gromov-Witten theory. We hope that the relation between knot theory and open Gromov-Witten theory will be explored much more in detail in the future. This will definitely open many new avenues for future research.

\section{References}

[1] M. Aganagic, A. Klemm, M. Mariño and C. Vafa, "The topological vertex", arxiv.org: hepth/0305132.

[2] A.K. Aiston and H.R. Morton, "Idempotents of Hecke algebra of type A", J. Knot theory Ramif. 7 (1998), 463-487.

[3] P. Freyd, D. Yetter, J. Hoste, W.B.R. Lickorish, K. Millet and A. Ocneanu, "A new polynomial invariant of knots and links", Bull. Amer. Math. Soc. 12 (1985) 239.

[4] R. Gopakumar and C. Vafa, "On the gauge theory/geometry correspondence", Adv. Theor. Math. Phys. 3 (1999) 1415.

[5] I.P. Goulden, A differential operator for symmetric functions and the combinatorics of multiplying transpositions, Trans. Amer. math. Soc. 344 (1994), 421.

[6] S. Gukov, "Three-dimensional quantum gravity, Chern-Simons theory, and the A-polynomial", Comm. Math. Phys. 255 (2005), no. 3, 577.

[7] A. Gyoja, "A q-analogue of Young symmetrisers", Oska J. Math. 23 (1996), 841.

[8] V.F.R Jones, "Hecke algebras representations of braid groups and link polynomials", Ann. of Math. 126 (1987) 335.

[9] L.H. Kauffman, "An invariant of regular isotopy", Trans. Amer. Math. Soc. 318 (1990) 417.

[10] J.M.F. Labastida and M. Mariño, "A new point of view in the theory of knot and link invariants", J. Knot Theory Ramif. 11 (2002), 173. 
[11] J.M.F. Labastida and M. Mariño, "Polynomial invariants for torus knots and topological strings", Comm. Math. Phys. 217 (2001), no. 2, 423.

[12] J.M.F. Labastida, M. Marino and C. Vafa, "Knots, links and branes at large N", J. High Energy Phys. 2000, no. 11, Paper 7.

[13] J. Li, C.-C. Liu, K. Liu and J. Zhou, "A mathematical theory of the Topological Vertex", math.AG/0408426.

[14] X.-S. Lin and H. Zheng, "On the Hecke algebra and the colored HOMFLY polynomial", math.QA/0601267.

[15] C.-C. Liu, K. Liu and J. Zhou, "A proof of a conjecture of Mariño-Vafa on Hodge integrals", J. Differential Geom. 65 (2003), no. 2, 289.

[16] C.-C. Liu, K. Liu and J. Zhou, "A formula of two-partition Hodge integrals", J. Amer. Math. Soc. 20 (2007), no. 1, 149.

[17] K. Liu and P. Peng, "Proof of the Labastida-Marino-Ooguri-Vafa Conjecture ", arXiv: 0704.1526.

[18] I. G. MacDonald, "Symmetric Functions and Hall Polynomials", 2nd edition, Clarendon Press, 1995.

[19] M. Mariño and C. Vafa, "Framed knots at large N", Orbifolds in mathematics and physics (Madison, WI, 2001), 185-204, Contemp. Math., 310, Amer. Math. Soc., Providence, RI, 2002.

[20] O. Ogievetsky and P. Pyatov, "Lecture on Hecke algebras", Lectures notes at the International School "Symmetries and Integrable Systems", Dubna, 1999.

[21] H. Ooguri and C. Vafa, "Knot invariants and topological strings", Nucl. Phys. B 577 (2000), 419.

[22] P. Peng, "A simple proof of Gopakumar-Vafa conjecture for local toric Calabi-Yau manifolds", to appear, Commun. Math. Phys.

[23] P. Peng, "Integrality structure in Gromov-Witten theory", Preprint.

[24] M. Rosso and V. Jones, "On the invariants of torus knots derived from quantum groups", J. Knot Theory Ramifications 2 (1993), 97.

[25] V. G. Turaev, "The Yang-Baxter equation and invariants of links", Invent. Math. 92 (1988), 527.

[26] V.A. Vassiliev, "Cohomology of knot spaces", Theory of singularities and its applications, Advances in Soviet Mathematics, vol. 1, Amer. Math. Soc., Providence, RI, 1990, 23.

[27] E. Witten, "Quantum field theory and the Jones polynomial", Commun. Math. Phys. 121 (1989) 351.

[28] E. Witten, "Chern-Simons gauge theory as a string theory", arxiv.org: hel-th/9207094, in The Floer memorial volume, The Floer memorial volume, 637, Progr. Math., 133, Birkhäuser, Basel, 1995.

Center of Mathematical Science, Zhejiang University, Box 310027, Hangzhou, China

E-mail address: liu@cms.zju.edu.cn

Department of Mathematics, UClA, Box 951555, Los Angeles, CA, 90095-1555

E-mail address: liu@math.ucla.edu

Department Of Mathematics, University of Arizona, 617 N. Santa Rita Ave, Tucson, $\mathrm{AZ}, 85721$.

E-mail address: ppeng@math.arizona.edu 